\title{
Plasma Levels of the Engulfment and Cell Motility Protein-1 are Associated with Kidney Damage in Diabetic Nephropathy: A Single-Center Pilot Study in Indonesia Population
}

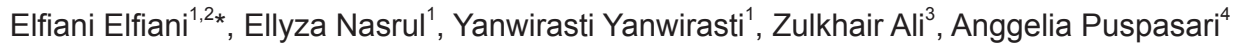 \\ ${ }^{1}$ Doctoral Degree of Biomedical Sciences, Faculty of Medicine, Universitas Andalas, Padang, Indonesia; ${ }^{2}$ Department of \\ Internal Medicine, Faculty of Medicine and Health Science, Universitas Jambi, Jambi, Indonesia; ${ }^{3}$ Department of Internal \\ Medicine, Faculty of Medicine, Universitas Sriwijaya, Palembang, Indonesia; ${ }^{4}$ Department of Biochemistry, Faculty of Medicine \\ and Health Science, Universitas Jambi, Jambi, Indonesia
}

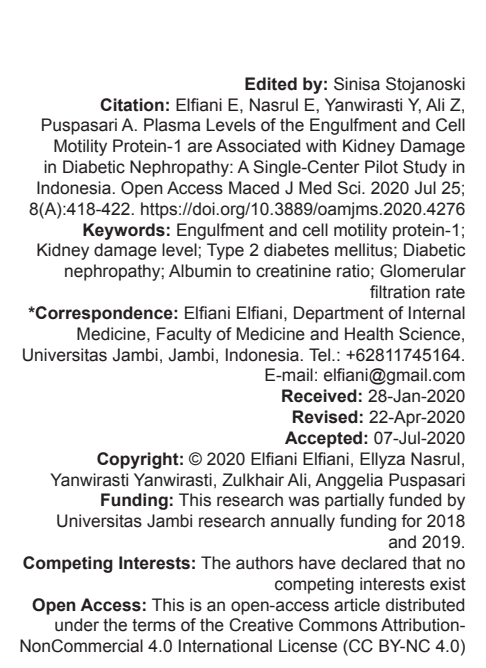

\section{Abstract}

BACKGROUND: Diabetic nephropathy (DN) is a chronic kidney disease with microvascular complications which influence a patient's quality of life and poses a potentially significant economic burden. The discovery of new biomarkers is needed to gain a better understanding and find additional treatment approaches beyond controlling plasma glucose and blood pressure. The engulfment and cell motility protein called engulfment and cell motility protein (ELMO)-1 is known to play a role in tissue remodeling and is associated with DN progression in animal models. Human studies correlating this protein with DN have been limited, although a genetic variation of ELMO-1 has been associated with DN.

AIMS: This research aimed to investigate the association and correlation of circulating ELMO-1 with the level of kidney damage in DN.

METHODS: The research design was cross-sectional. In this study, 60 patients with type 2 diabetes mellitus (T2DM) participated. Patients' blood pressure, plasma glucose, and renal function parameters were measured to confirm the diagnosis. Albumin to creatinine ratio (ACR) was used to assess the level of kidney damage and determines DN group.

RESULTS: Analyses shown the severity of albuminuria which is associated with plasma ELMO-1 when DN is evident. Furthermore, in all study participants, plasma ELMO-1 was correlated with increased proteinuria, ACR, and decline of glomerular filtration rate.

CONCLUSIONS: Our research findings provide support for ELMO-1 as a promising marker of kidney damage leve in patients with T2DM, especially when DN has occurred. Larger sample sizes and multicenter research in the Indonesian population are needed to confirm these results.

\section{Introduction}

The incidence of diabetic nephropathy (DN) has increased worldwide, especially in Asian populations. This condition leads to end-stage renal diseases which often decrease a patient's quality of life and cause potentially significant economic burden [1], [2]. Additional approaches are needed to prevent and treat DN beyond controlling hyperglycemia and high blood pressure. Research of new biomarkers is important to gain a better understanding of DN progression and further develop new treatment approaches to prevent the progression of DN [3], [4], [5].

Increased microalbuminuria and decreased glomerular filtration rate (GFR) are clinical markers of DN [6]. Tissue remodeling, including alteration of matrix deposition, podocytes, and endothelial injury, which increases cell apoptosis, may happen before clinical markers of DN are detected [7]. These events are associated with alteration of some circulating protein expressions, which are promising as new biomarkers before the commonly used clinical markers of DN appear [3], [7], [8].

The engulfment and cell motility protein called engulfment and cell motility protein (ELMO)-1 is known to play a role in tissue remodeling. The exact role of this protein in human DN pathophysiology is still unknown, although some genetic association studies reported that the ELMO-1 gene is a strong candidate as a DN associated gene. Polymorphism in the intronic region of ELMO-1 was associated with DN in Asia and America populations. None have described any association of its polymorphisms or DN with systemic or local ELMO-1 expression, although translation studies in animal models have reported that the ELMO-1 protein does play a role in the progression of DN [9], [10], [11], [12].

Diabetic rat models which have different systemic levels of ELMO-1 shown higher ELMO-1 expression that was associated with severity of renal fibrosis and amount 
of urinary albumin excretion through aggravated lipid peroxidation and increased tumor growth factor beta-1 (TGF $\beta$-1) levels, which are both markers of extracellular matrix expansion [13]. Another animal translation study that used a genetically modified zebrafish model shown that ELMO-1 is an important factor for glomerular protection and renal cell survival through decreasing apoptosis, especially under diabetic conditions [14].

This research was conducted because studies in humans about ELMO-1 expression in DN are limited, and recent findings in animal models briefly describe the promising potential of ELMO-1 as a new biomarker which plays a role in DN progression. This research aimed to investigate the association and correlation of circulating ELMO-1 in the progression of DN in the Indonesian population for the $1^{\text {st }}$ time.

\section{Materials and Methods}

\section{Study design}

This research was a cross-sectional study. A total of 60 patients with type II diabetes mellitus (T2DM) were recruited from the Jambi province who fulfilled eligible criteria for this research. Inclusion criteria were as follows: Patient with T2DM who was suffering from DM for at least 5 years, with an age range of 30-60 years old. We excluded patients with urinary infections based on clinical examination and routine urine analysis, history of other kidney diseases based on the medical record, pregnant women, and immunocompromised subjects. Patients' T2DM diagnosis was based on fasting plasma glucose $>126 \mathrm{mg} / \mathrm{dL}$ and $2 \mathrm{~h}$ postprandial plasma glucose $>200 \mathrm{mg} / \mathrm{dL}$, as listed in medical records. In all patients, we measured albumin to creatinine ratio (ACR), GFR, and plasma creatinine as kidney function parameters and plasma ELMO-1. We also measured blood pressure and plasma glucose levels. All research subjects signed informed consent forms after receiving a detailed explanation about the study objectives and design. The research protocol was arranged based on the Helsinki Declaration and was approved by the research ethics committee of the Faculty of Medicine, Andalas University, with approval registration number 706/KEP/FK/2019.

\section{Kidney function parameters}

Random spot urine samples as much as $30 \mathrm{~mL}$ were collected from the patients. Quantitative measurements of urine albumin were made with immunoturbidimetric. Urine creatinine was measured with enzymatic colorimetric. Then, the ACR was calculated based on the ratio of quantitative creatinine to albumin in random spot urine sampling. DN was diagnosed according to ACR, where DN was defined as $A C R \geq 30 \mathrm{mg} / \mathrm{g}$. Furthermore, we also grouped degree of kidney damage level according to ACR, with normal to mildly albuminuria as the $A 1$ group ( $A C R<30 \mathrm{mg} / \mathrm{g}$ ), the moderately increased albuminuria as the A2 group (ACR $30-300 \mathrm{mg} / \mathrm{g}$ ), and the severely increased albuminuria as the A3 group (ACR >300 mg/g). GFR was calculated based on chronic kidney disease-epidemiology collaboration equation, based on estimated creatinine serum, gender equation coefficient, and age. Plasma creatinine levels were also measured with enzymatic colorimetric.

\section{Measurement ELMO-1}

Blood samples as much as $5 \mathrm{ml}$ were collected from the antecubital vein. The centrifuge was performed within 30 min after collection and samples stored in $-20^{\circ} \mathrm{C}$ before performing ELISA. Sandwich ELISA protocol for human ELMO-1 was used to measure ELMO-1 (MyBiosource ${ }^{R}$ ) with catalog number MBS9321199. Random twice repetition of ELISA for some samples was used to confirm the results of ELISA.

\section{Results}

\section{Baseline subject characteristics}

Baseline subject characteristics are shown in Table 1. A total of 60 subjects who fulfilled the research inclusion criteria participated in this study, with 20 subjects in each group.

Demographic characteristics shown that the mean age was younger in the $\mathrm{A} 1$ group than the other groups. Female subjects were more frequent in the A1 and A2 groups, but it was not statistically significant.

We measured kidney function for each subject. The A1 group had lower urine creatinine, but it was not statistically significant. Lower plasma creatinine, urine albumin, ACR, and higher GFR were found in the A1 group compared to the other groups, and the differences were statistically significant. These baseline subject characteristics shown that the A1 (non-ND) group had better kidney function than the A2 and A3 groups (ND).

All patients had been diagnosed with T2DM. The A1 group had lower fasting and postprandial plasma glucose than the $A 2$ and $A 3$ groups, but it was not statistically significant. Lower systolic and diastolic blood pressures were also found in the A1 group compared to the other groups, and the result was statistically significant.

Plasma ELMO-1 level was associated with the severity of kidney level damage based on ACR when DN occurs

The mean of ELMO-1 plasma level based on the grade of ACR is shown in Figure 1. ELMO-1 plasma 
Table 1: Baseline subject characteristic

\begin{tabular}{|c|c|c|c|c|}
\hline Characteristic & $\mathrm{A} 1(\mathrm{n}=20)$ & $\mathrm{A} 2(\mathrm{n}=20)$ & $\mathrm{A} 3(\mathrm{n}=20)$ & $p$-value \\
\hline Age and years old & $48.05 \pm 9.09$ & $50.35 \pm 7.36$ & $51.95 \pm 7.64$ & $0.314^{\star *}$ \\
\hline Gender & & & & 0.619 \\
\hline Male, $\mathrm{n}$ & 8 & 7 & 10 & \\
\hline Female, $\mathrm{n}$ & 12 & 13 & 10 & \\
\hline Fasting plasma glucose, $\mathrm{mg} / \mathrm{dL}$ & $140.95 \pm 35.49$ & $154.80 \pm 53.98$ & $164.95 \pm 57.69$ & $0.513^{*}$ \\
\hline Postprandial plasma glucose, $\mathrm{mg} / \mathrm{dL}$ & $242.7 \pm 96.09$ & $224.55 \pm 50.66$ & $257.10 \pm 57.79$ & $0.321^{*}$ \\
\hline Systolic blood pressure, $\mathrm{mmHg}$ & $120.00(100.00-160.00)$ & $130.00(110.00-150.00)$ & $150.00(110.00-170.00)$ & $<0.001^{\text {** }}$ \\
\hline Diastolic blood pressure, $\mathrm{mmHg}$ & $80.00(50.00-90.00)$ & $80.00(60.00-90.00)$ & $80.00(70.00-100.00)$ & $0.042^{* *}$ \\
\hline Plasma creatinine, $\mathrm{mg} / \mathrm{dL}$ & $0.88 \pm 0.38$ & $0.90 \pm 0.22$ & $1.92 \pm 1.06$ & $0.001^{*}$ \\
\hline Urine creatinine, $\mathrm{mg} / \mathrm{dL}$ & $86.21(13.43-377.97)$ & $114.04(19.69-351.55)$ & $79.31(11.43-192.99)$ & $0.217^{\star *}$ \\
\hline Urine albumin, $\mathrm{mg} / \mathrm{dL}$ & $14.7 \pm 10.77$ & $89.25 \pm 83.71$ & $2015.80 \pm 2755.93$ & $<0.001^{*}$ \\
\hline Albumin creatinine ratio & $12.82(4.06-28.44)$ & $47.36(30.68-203.77)$ & $2263.00(349.96-14403.00)$ & $<0.001^{* *}$ \\
\hline Glomerular filtration rat, $\mathrm{mL} / \mathrm{min}$ & $92.66 \pm 23.59$ & $84.17 \pm 22.55$ & $50.34 \pm 37.10$ & $<0.001^{*}$ \\
\hline
\end{tabular}

level for each group was not normally distributed even after transformation with $\log 10$, so the non-parametric test was performed. The mean value of the ELMO-1 plasma level of the $\mathrm{A} 1$ group was lower than the mean value of ELMO-1 plasma level of $A 2$, but it was not statistically significant $(p=0.779)$. With the limited number of samples, this result has shown that ELMO-1 was not associated with the level of kidney damage of microalbuminuria (A1 group) to early macroalbuminuria (A2 group).

Table 2: ELMO-1 level based on clinical characteristics affected by DN

\begin{tabular}{ll}
\hline Group parameter & ELMO-1 level \\
\hline Glomerular filtration rate & \\
$\quad$ GFR $\geq 60 \mathrm{~mL} / \mathrm{min}, \mathrm{n}=41$ & $134.22(29.10-3726.78)$ \\
$\quad \mathrm{GFR}<60 \mathrm{~mL} / \mathrm{min}, \mathrm{n} 19$ & $128.54(61.77-3446.93)$ \\
Fasting plasma glucose & \\
$\quad \mathrm{FPG} \leq 130 \mathrm{mg} / \mathrm{dL}, \mathrm{n}=25$ & $121.44(33.36-3446.93)$ \\
$\mathrm{FPG}>130 \mathrm{mg} / \mathrm{dL}, \mathrm{n}=35$ & $134.22(29.10-3726.78)$ \\
$2 \mathrm{~h}$ postprandial plasma glucose & \\
$2 \mathrm{~h} \mathrm{pp} \leq 180 \mathrm{mg} / \mathrm{dL}, \mathrm{n}=9$ & $144.16(94.45-3131.57)$ \\
$2 \mathrm{~h} p \mathrm{p}>180 \mathrm{mg} / \mathrm{dL}, \mathrm{n}=51$ & $128.54(29.10-3726.78)$ \\
Blood pressure & \\
$\mathrm{SBP}<140 \mathrm{mmHg}$ and DBP $<90 \mathrm{mmHg}, \mathrm{n}=26$ & $134.22(29.10-1976.67)$ \\
$\mathrm{SBP} \geq 140 \mathrm{mmHg}$ and or DBP $\geq 90 \mathrm{mmHg}, \mathrm{n}=34$ & $126.41(61.77-3726.78)$ \\
\hline
\end{tabular}

The mean value of ELMO-1 plasma level of A1 group was lower than the mean value of ELMO-1 plasma level of $A 3$, and it was statistically significant ( $p$ $=0.005$ ). The mean value of ELMO-1 plasma level of the A2 group was lower than mean value of ELMO-1 plasma level of the A3 group, and it was statistically significant $(p=0.009)$. This result shown that ELMO-1 plasma level was associated with level of kidney damage based on ACR when DN has occurred (A2 and A3 groups were confirmed DN).

Table 3: Correlation of ELMO-1 with clinical parameter

\begin{tabular}{lll}
\hline Kidney function & \multicolumn{2}{l}{ Serum ELMO-1 $(\mathrm{ng} / \mathrm{mL})$} \\
\cline { 2 - 3 } & $\mathrm{R}$ & $\mathrm{p}$-value \\
\hline Plasma creatinine, $\mathrm{mg} / \mathrm{dL}$ & 0.078 & 0.554 \\
Urine creatinine, $\mathrm{mg} / \mathrm{dL}$ & -0.100 & 0.447 \\
Urine albumin, $\mathrm{mg} / \mathrm{dL}$ & 0.344 & 0.007 \\
Fasting plasma glucose, $\mathrm{mg} / \mathrm{dL}$ & 0.013 & $0.919^{*}$ \\
Postprandial plasma glucose, $\mathrm{mg} / \mathrm{dL}$ & 0.110 & 0.403 \\
Systolic blood pressure, $\mathrm{mmHg}$ & 0.152 & 0.245 \\
Diastolic blood pressure, $\mathrm{mmHg}$ & 0.043 & 0.745 \\
Glomerular filtration rate, $\mathrm{mL} / \mathrm{min}$ & -0.293 & $0.023^{*}$ \\
Albumin creatinine ratio & 0.383 & 0.003 \\
Age, years old & 0.197 & $0.132^{*}$ \\
\hline${ }^{*}$ Pearson correlation test was performed due to normally distributed data of one variable. Otherwise, the \\
Spearman correlation was performed.
\end{tabular}

Table 2 shows that the mean value of ELMO-1 plasma level was higher in variables affected by DN. Higher plasma ELMO-1 levels were found in the lower
GFR group, in higher plasma glucose fasting and $2 \mathrm{~h}$ postprandial plasma glucose and higher blood pressure groups. Although plasma ELMO-1 level difference were not significant statistically between these groups.

\section{Plasma ELMO-1 level was correlated with kidney function in all subjects with T2DM}

That correlations of ELMO-1 with some clinical parameters which play a role in the progression of DN are shown in Table 3. Urine albumin and ACR have a positive weak correlation with ELMO-1 plasma level, and the result was statistically significant. GFR had a negative weak correlation with ELMO-1 plasma level, and the result was statistically significant.

Plasma creatinine, plasma glucose, blood pressure, and age had very weak positive correlations with ELMO-1 plasma level. Urine creatinine had a very weak negative correlation with ELMO-1 plasma level. This correlation was not statistically significant.

\section{Discussion}

DN, also known as diabetic kidney disease, causes structural changes and declining kidney function. Albuminuria and GFR are clinical markers widely used to monitor the progression of DN. Both markers are endpoints of many pathways, so prevention of decreases of the two markers is not easy. Identifying additional new markers which are directly involved in certain dominant pathways can assist in gaining better understanding of DN pathophysiology and help develop new approaches in DN treatment [3], [4], [5].

Analyses in our research shown the severity of albuminuria were associated with plasma ELMO-1 when DN was evident (Figure 1). Furthermore, in all study, plasma ELMO-1 was correlated with declining of kidney function in patients with T2DM based on levels of proteinuria, ACR, and GFR (Table 2). This finding demonstrates that the ELMO-1 plasma level is 
a promising biomarker of the severity level of kidney damage in DN in the Indonesia population. In addition, ELMO-1 plasma level is also a promising marker of declining kidney function in patients with T2DM.

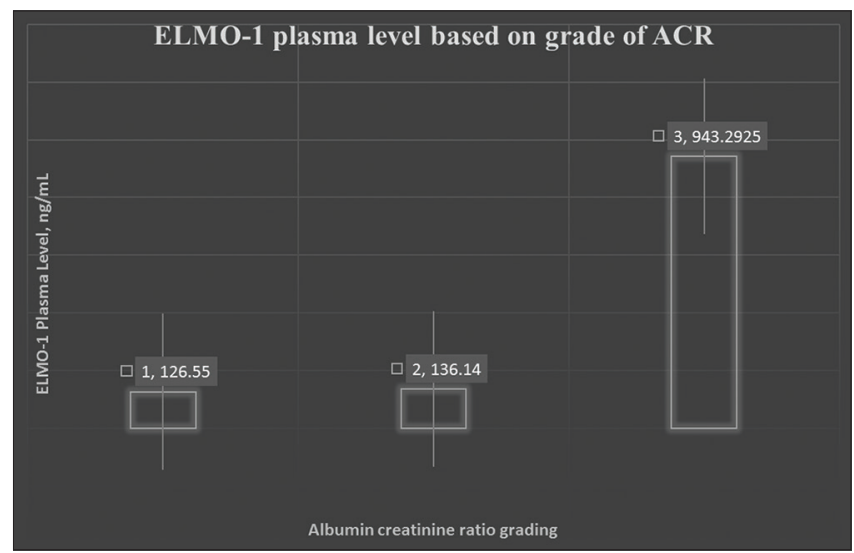

Figure 1: Mean of engulfment and cell motility protein-1 plasma level based on the grade of albumin to creatinine ratio

Studies of ELMO-1 plasma or tissue specifically associated with diabetes or DN in humans are still limited. One study found kidney expression of ELMO-1 in the non-diabetic compared to diabetic patient remained unchanged in podocytes and tubules, but it did not define whether the diabetic patient was suffering $D N$ or not [14]. Phenotype genotype association studies have shown that the ELMO-1 gene is a strong gene candidate associated with DN. Single nucleotide polymorphism in the intronic region of ELMO-1 gene was associated with DN in Asia and American populations, but none described the correlation with systemic or local ELMO-1 expression [9], [10], [11], [12].

One supporting study about the role of ELMO-1 related to DN described the association using an animal model. Using a zebrafish animal model, the results shown that ELMO-1 knockout model has a more detrimental effect on pronephric structure and function under hyperglycemia conditions compared to controls. This finding related to the detrimental effect on the processes of podocytes and glomerular ultrafiltration. Injection of ELMO-1 mRNA to create ELMO-1 overexpression and pan-caspase inhibitor rescue altered the renal structure and function. This research shown that ELMO-1 was an important factor for glomerular protection and renal cell survival that decreasing apoptosis, especially under diabetic conditions [14]. Another study found that a rat model with a mutation of in insulin-2 gene had different levels of EIMO-1. The research reported that higher ELMO-1 expression was associated with the severity of renal fibrosis and amount of urinary albumin excretion. The increased expression of ELMO-1 was followed by the aggravated marker of oxidative stress and $\mathrm{NAD}(\mathrm{P}) \mathrm{H}$ oxidase 4 and reduced glutathione, and the aggravated markers of fibrogenesis, TGF $\beta-1$, and endothelin-1 [13].

Baseline subject characteristics shown that blood mean systolic and diastolic blood pressures were associated with the degree of albuminuria, but plasma glucose was not, although lower plasma glucose was found in the A1 group compared to the A2 and $A 3$ groups. The previous study shown controlling glucose plasma and blood pressure influences the progression of DN, although the duration of diabetes also contributes to DN [2], [6], [7]. Age and gender were not confounding factors for this research, and in the baseline characteristics, it did not differ statistically between the three groups.

Random spot urine sampling for ACR is used as one of the markers of DN progression. In this research, we used this marker as a reference to know DN progression because a previous study reported that it was representative and more practical to assess proteinuria related to diabetic complications than 24-h urine samples. In the next step, we grouped ACR into three groups. There were A1 or mildly albuminuria, A2 or moderately increased or microalbuminuria, and $A 3$ or severe increased or macro-albuminuria. DN was confirmed and diagnosed with ACR in groups A2 and A3 [7], [15]. To the best of our knowledge, this is the first study of the association of circulating ELMO-1 with DN in the Indonesia population. Some limitations of our study were that it was conducted in only one center and had a limited number of participants which reduced the generalizability of the findings. To confirm the finding of ELMO-1 as a progression marker of severity in DN, further multicenter studies with larger numbers of participants are needed.

\section{Conclusions}

Plasma ELMO-1 level is associated with the severity level of kidney damage based on ACR when DN has occurred. Albuminuria and ACR had positive correlations with plasma ELMO-1 and GFR had a negative correlation with plasma ELMO-1 in all subjects who suffered T2DM. These findings shown that plasma ELMO-1 is a promising marker of the level of kidney damage, especially when DN has occurred in the Indonesia population. According to the limitations of this study, larger numbers of subjects and multicenter research are needed.

\section{Acknowledgment}

This study was a part of the author's Master thesis. The authors would like to acknowledge the contribution of staff at the biotechnology laboratory of the Medical Faculty University of Andalas, Prodia laboratory and Genetica Science laboratory, for their help in laboratory measurements. 


\section{References}

1. Koye DN, Magliano DJ, Nelson RG, Pavkov ME. The global epidemiology of diabetes and kidney disease. Adv Chronic Kidney Dis. 2018;25(2):121-32. https://doi.org/10.1053/j. ackd.2017.10.011 PMid:29580576

2. Gheith O, Farouk N, Nampoory N, Halim MA, Al-Otaibi T. Diabetic kidney diseases. World wide difference of prevalence and risk factors. J Nephropharmacol. 2016;5(1):49-56.

PMid:28197499

3. Persson F, Rossing P. Diagnosis of diabetic kidney disease: State of the art and future perspective. Kidney Int Suppl. 2018;8(1):2-7.

PMid:30675433

4. Lee C, Lam KS. Biomarkers of progression in diabetic nephropathy: The past, present and future. J Diabetes Investig. 2015;6(3):247-9. https://doi.org/10.1111/jdi.12329

PMid:25969706

5. Currie G, Mckay G, Delles C. Biomarkers in diabetic nephropathy: Present and future. World J Diabetes. 2014;5(6):763-76. PMid:25512779

6. Alicic RZ, Rooney MT, Tuttle KR. Diabetic kidney disease: Challenges, progress and possibilities. Clin J Am Soc Nephrol. 2017;12(12):2032-45. https://doi.org/10.2215/cjn.11491116 PMid:28522654

7. Fioretto $P$, Mauer M. Histopathology of diabetic nephropathy Semin Nephrol. 2007;27(2):195-207. https://doi.org/10.1016/j. semnephrol.2007.01.012 PMid: 17418688

8. Batlle D. Clinical and cellular markers of diabetic nephropathy. Kidney Int. 2003;63(6):2319-30. https://doi. org/10.1046/j.1523-1755.2003.00053.x

PMid:12753325
9. Shimazaki A, Kawamura Y, Kanazawa A, Sekine A, Saito S, Tsunoda T, et al. Genetic variations in the gene encoding ELMO1 are associated with susceptibility to diabetic nephropathy. Diabetes. 2005;54(4):1171-8. https://doi.org/10.2337/ diabetes.54.4.1171

PMid: 15793258

10. Pezzolesi MG, Katavetin P, Kure M, Poznik GD, Skupien J, Mychaleckyj JC, et al. Confirmation of genetic associations at ELMO1 in the GoKinD collection supports its role as a susceptibility gene in diabetic nephropathy. Diabetes. 2009;58(11):2698-702. https://doi.org/10.2337/db09-0641 PMid:19651817

11. Bodhini D, Chidambaram M, Liju S, Revathi B, Laasya D, Sathish N, et al. Association of rs11643718 SLC12A3 and rs741301 ELMO1 variants with diabetic nephropathy in South Indian population. Ann Hum Gen. 2016;80(6):1-6. https://doi. org/10.1111/ahg.12174

PMid:27699784

12. Mehrabzadeh $M$, Pasalar $P$, Karimi $M$, Abdollahi $M$, Daneshpour M, Asadolahpour E, et al. Association between ELMO1 gene polymorphisms and diabetic nephropathy in an Iranian population. J Diabetes Metab Disord. 2016;15:43. https://doi.org/10.1186/s40200-016-0265-3

PMid:27761430

13. Hathaway CK, Chang AS, Grant R, Kim H, Madden VJ Bagnell CR, et al. High Elmo1 expression aggravates and low Elmo1 expression prevents diabetic nephropathy. Proc Natl Acad Sci U S A. 2016;113(8):2218-22. https://doi.org/10.1073/ pnas. 1600511113 PMid:26858454

14. Sharma KR, Heckler K, Stoll SJ, Hillebrands J, Kynast K, Herpel E, et al. ELMO1 protects renal structure and ultrafiltration in kidney development and under diabetic conditions. Sci Rep. 2016;6:37172. https://doi.org/10.1038/srep37172

15. Roshan B, Stanton RC. A story of microalbuminuria and diabetic nephropathy. J Nephropathol. 2013;2(4):234-40.

PMid:24475455 